\title{
VLBA polarimetric observations of the CSS quasar 3C 147
}

\author{
A. Rossetti ${ }^{1}$, F. Mantovani ${ }^{1}$, D. Dallacasa ${ }^{1,2}$, W. Junor ${ }^{3}$, C. J. Salter ${ }^{4}$, and D. J. Saikia ${ }^{5}$ \\ 1 Istituto di Radioastronomia - INAF, via Gobetti 101, 40129, Bologna, Italy \\ e-mail: rossetti@ira.inaf.it \\ 2 Dipartimento di Astronomia, Università degli Studi, via Ranzani 1, 40127 Bologna, Italy \\ 3 Los Alamos National Laboratory, Los Alamos, NM 87545, USA \\ 4 Arecibo Observatory, HC3 Box 53995, Arecibo 00612, Puerto Rico \\ 5 National Centre for Astrophysics, TIFR, Post Bag 3, Ganeshkhind, Pune 411 007, India
}

Received 20 October 2008 / Accepted 24 April 2009

\section{ABSTRACT}

\begin{abstract}
Aims. We report new VLBA polarimetric observations of the compact steep-spectrum (CSS) quasar 3C 147 (B0538 + 498) at 5 and $8.4 \mathrm{GHz}$.

Methods. By using multifrequency VLBA observations, we derived milliarcsecond-resolution images of the total intensity, polarisation, and rotation measure distributions, by combining our new observations with archival data.

Results. The source shows a one-sided structure, with a compact region, and a component extending about 200 mas to the south-west. The compact region is resolved into two main components with polarised emission, a complex rotation measure distribution, and a magnetic field dominated by components perpendicular to the source axis.

Conclusions. By considering all the available data, we examine the possible location of the core component, and discuss two possible interpretations of the observed structure of this source: core-jet and lobe-hot spot. Further observations to unambiguously determine the location of the core would help distinguish between the two possibilities discussed here.
\end{abstract}

Key words. polarization - galaxies: quasars: individual: 3C 147 - galaxies: jets - radio continuum: galaxies

\section{Introduction}

Compact steep-spectrum (CSS) radio sources are scaleddown versions of large-sized double sources with linear sizes $\leq 20 h^{-1} \mathrm{kpc}^{1}$ and steep high-frequency radio spectra $\left(\alpha>0.5^{2}\right)$. It is now generally believed that most CSS sources are young, of ages $<10^{3-5} \mathrm{yr}$, whose radio lobes have had insufficient time to grow to kilo-parsec scales (Fanti et al. 1995). Subgalactic in size, they reside deep inside their host galaxies and are largely confined to the narrow line region (NLR) with its relatively large column density of ionised plasma. This magnetised thermal plasma has different indices of refraction for the two circular polarised modes of the synchrotron emission of these sources. This rotates the orientation of the linearly polarised components of the transmitted radiation. The amount of the rotation is given by $\Delta \chi=812 \times \lambda^{2} \int n_{\mathrm{e}} B_{\|} \mathrm{d} \ell$, where $\lambda$ is the wavelength in $\mathrm{cm}$, $n_{\mathrm{e}}$ is the electron density of the medium in $\mathrm{cm}^{-3}, B_{\|}$is the component of the magnetic field along the line of sight in $\mu \mathrm{G}$, and $\ell$ is the geometrical depth of the medium along the line of sight in kpc.

Since the rotation is proportional to $\lambda^{2}$, it can be determined from observations at a number of wavelengths. The Faraday rotation measure, $R M$, is the amount of rotation expressed in $\mathrm{rad} \mathrm{m}^{-2}$. Even for moderate magnetic field intensity for the foreground screen, significant Faraday rotation effects external to the radio sources are to be expected, which can completely depolarise sources at long wavelengths. The comparison of polarisation images over a range of wavelengths, preferably with similar resolutions, is then an important diagnostic of the physical

\footnotetext{
${ }^{1} q_{0}=0.5$ and $H_{0}=100 h \mathrm{~km} \mathrm{~s}^{-1} \mathrm{Mpc}^{-1}$.

2 We assume $S_{v}=v^{-\alpha}$.
}

conditions within and around CSS radio sources. CSS radio galaxies show very low, or even no, linear polarisation at centimetre wavelengths. CSS quasars are instead found to have polarisation percentages as high as $10 \%$ above $1 \mathrm{GHz}$ (Saikia et al. 1985, 1987; Fanti et al. 2004; Rossetti et al. 2008).

A minority of CSSs exhibit complex or highly-asymmetric structures. Observations indicate that intrinsic distortions are caused by interactions with a dense, inhomogeneous gaseous environment (Mantovani et al. 1994). This view is supported by the most distorted and complex structures being found in jet-dominated objects with very weak cores (Mantovani et al. 2002). Furthermore, an increased asymmetry in terms of intensity, arm ratio, spectral index, and polarisation with decreasing source linear size suggests that they are expanding through the dense inhomogeneous interstellar medium (ISM) of their host galaxies (Sanghera et al. 1995; Saikia et al. 2001; Saikia \& Gupta 2003; Rossetti et al. 2006).

Sub-arcsec polarimetry provided evidence of the interaction of components of CSS sources with dense clouds of gas (Nan et al. 1999). According to Junor et al. (1999a), the CSS source 3C 147 has the expected signatures of a jet colliding with a cloud of gas on the southwestern side of the galaxy. Optical observations also show evidence of such interactions. Gelderman \& Whittle (1994) found that CSS sources have relatively strong, high equivalent width, high excitation line emission, with broad, structured [OIII] $\lambda 5007$ profiles, which they interpret as evidence of strong interaction between the jet and the ISM.

The number of CSSs for which detailed radio polarisation information is available remains small. To improve the statistics, we are conducting a series of observations to image examples with moderate degrees of polarised emission and clear signatures of interaction with their environment, namely fractional 
polarisations that decrease with increasing wavelength, and $R M>450 \mathrm{rad} \mathrm{m}^{-2}$ in the source rest frame. Results for the first two CSS quasars observed in this ongoing program, B0548+165 and B1524-136, are available in Mantovani et al. (2002).

We present results for 3C $147(\mathrm{~B} 0538+498)$, imaged with milliarcsecond resolution via full-Stokes VLBA observations. This CSS radio source is associated with a QSO of $m_{v}=16.9$ and $z=0.545$ (Spinrad et al. 1985). It has a total angular extent of 0.6 , corresponding to a projected linear size of $\approx 2.2 \mathrm{~h}^{-1} \mathrm{kpc}$. $3 C 147$ shows a very large integrated $R M$ of $\approx-1500 \mathrm{rad} \mathrm{m}^{-2}$ in the observer's frame (Inoue et al. 1995), implying that the radio source is surrounded by a dense ionised medium. On the sub-arcsecond scale 3C 147 exhibits a very asymmetric doublelobed radio structure with a strong, steep-spectrum, central component and asymmetric components of even steeper spectrum situated to the southwest and north (Akujor \& Garrington 1995; Lüdke et al. 1998; Junor et al. 1999a). An HST optical study of the emission-line region of CSS radio sources (Axon et al. 2000) shows that the emission-line morphology ([OIII] $\lambda$ 5007) of 3C 147 is dominated by an unresolved central component, embedded in a more diffuse region, which extends over $\approx 1$ 1.'2 and is well aligned with the double radio structure observed on the sub-arcsecond scale.

Junor et al. (1999a) imaged the polarised emission of 3C 147 using A-array VLA observations at $X$ - and $U$-bands. They measured significant differential Faraday rotation between the northern and the main (central+south-western) components situated on opposite sides of the quasar, and a strong east-west gradient in the polarisation percentage of the main component, whose emission depolarises strongly towards lower frequencies. They interpreted these results in terms of an asymmetric environment with large amounts of thermal gas on the western side.

Very long baseline interferometry (VLBI) observations have been analysed to indicate that the central component contains a compact core with a jet emerging from it toward the southwest (Alef et al. 1990; Nan et al. 2000). Part of this jet yields evidence of mild superluminal motion: Alef et al. (1990) reported $\approx 1.3 c$, and Nan et al. (2000) inferred $\approx 1.5 c$.

In this paper, we present multi-frequency VLBA (plus a single VLA antenna) polarisation observations at 5 and $8.4 \mathrm{GHz}$. In Sect. 2 we summarise the observations and data processing. Section 3 describes new information obtained about the morphological and polarisation properties of 3C 147. Discussion and conclusions are presented in Sects. 4 and 5.

\section{Observations and data reduction}

\section{1. $5 \mathrm{GHz}$ and $8.4 \mathrm{GHz}$ data}

Polarimetric observations of 3C 147 were carried out in May 2001 at 5 and $8.4 \mathrm{GHz}$ for about $12 \mathrm{~h}$ with the VLBA plus one VLA antenna. This array has the highest quality observing capability for detecting the extended, complex, milliarcsecond structure of this source. The data were recorded in both right- and left-circular polarisation in four $8-\mathrm{MHz}$ bands at each frequency. At $5 \mathrm{GHz}$, the observations were spread across the available bandwidth of $\approx 500 \mathrm{MHz}$, allowing us to obtain four truly simultaneous and independent polarisation images and study the rotation measure distribution. To increase the sensitivity to the polarised emission, at $8.4 \mathrm{GHz}$ we decided to use contiguous IFs. The resulting Intermediate Frequencies (IFs) are $4619 \mathrm{MHz}, 4657 \mathrm{MHz}, 4854 \mathrm{MHz}$, and $5094 \mathrm{MHz}$ at $C$ band, and $8405 \mathrm{MHz}, 8413 \mathrm{MHz}, 8421 \mathrm{MHz}$, and $8429 \mathrm{MHz}$ at $X$ band. The data were correlated with the NRAO VLBA processor at Socorro and calibrated, imaged, and analysed using the AIPS package.

After the application of system temperature and antenna gain information, the amplitudes were checked by means of the data on the source DA 193, which was used as flux density calibrator, fringe finder, and polarisation calibrator. All the gain corrections were found to be within the $3 \%$, which can be conservative assumed as absolute calibration uncertainty. Standard procedures were applied for the fringe fitting of both total intensity and polarised signal.

During the data reduction process, the data at each of the four IFs within the $C$-band were processed separately. The polarised source DA 193 was used to determine the instrumental polarisation ("D-term") using the AIPS task PCAL. The solution shows that the instrumental polarisation was typically of the order of $1 \%$. After the D-term calibration, there remains an arbitrary offset in the polarisation position angle, $\chi$, which can be determined by using integrated measurements of the source DA 193 from the NRAO polarisation database, assuming that the source emission is confined to within its milliarcsecond structure. Since DA 193 exhibits time variability and there were no NRAO measurements close to our observing epoch (2001 May 6), we had to extrapolate the polarisation of DA 193 using a linear interpolation of all the measurements available for this source from 2000 to 2002 at the VLA/VLBA polarisation calibration page ${ }^{3}$. To remove any offsets between the four IFs at $C$-band, we first rotated the phases of the polarisation $u v$ data for all sources so that the $\chi$ values for DA 193 at each IF were aligned with the value of IF1 $(4619 \mathrm{MHz})$. This procedure assumed that the Faraday rotation of DA 193 across the observing bandwidth was negligible, a reasonable assumption given the low integrated $R M$ of this source $\left(\approx 107 \mathrm{rad} \mathrm{m}^{-2}\right)$. A systematic error of $\approx 5^{\circ}$ was introduced over the whole bandwidth. As a result, there is an uncertainty in the $R M$ within the $C$-band. As an overall check of the reliability of our calibration process, we compared the results obtained for the source $0927+390$ with those found in the NRAO data archive. The polarisation angles derived at $4.8 \mathrm{GHz}\left(\chi=61^{\circ} 7\right)$ and $8.4 \mathrm{GHz}$ (71.3) from our calibration procedure are very close to the NRAO measurements made 17 days before $\left(\chi=58^{\circ} .3\right.$ at $4.8 \mathrm{GHz}$ and $\chi=68.5$ at $8.4 \mathrm{GHz})$ and 30 days after $\left(\chi=58^{\circ} .7\right.$ at $4.8 \mathrm{GHz}$ and $\chi=70^{\circ} .9$ at $8.4 \mathrm{GHz}$ ) our observing epoch. This confirms that our calibrations of the electric vector polarisation angles are accurate to within about $5^{\circ}$.

To derive $R M$ information, images were obtained at the different frequencies by using the $u v$-range between the shortest baseline at the highest frequency and the longest baseline at the lowest frequency, and convolving to the lowest resolution. Then, a data cube in $\lambda^{2}$ at four (the maximum number of frequencies accepted by the AIPS task RM) observed frequencies (4619, 4854, 5094, and $8421 \mathrm{MHz}$ ) was obtained using the AIPS task MCUBE. The AIPS task RM was used to produce a weighted fit of the observed $\chi$ values to a $\lambda^{2}$ dependence. Finally, the intrinsic magnetic-field directions were obtained using the derived $R M$ distribution to de-rotate the observed $\chi$ values for the VLBI polarisation distribution.

\subsubsection{Archival data}

We also reanalysed data from projects BC033 (May 1995; Nan et al. 2000) and BZ0023 (August 2000; Zhang et al. 2004) at $C$ - and $X$-bands, respectively, to obtain a more accurate determination of $R M$. The $C$-band observations by Nan et al. (2000)

3 http://www.vla.nrao.edu/astro/calib/polar/ 


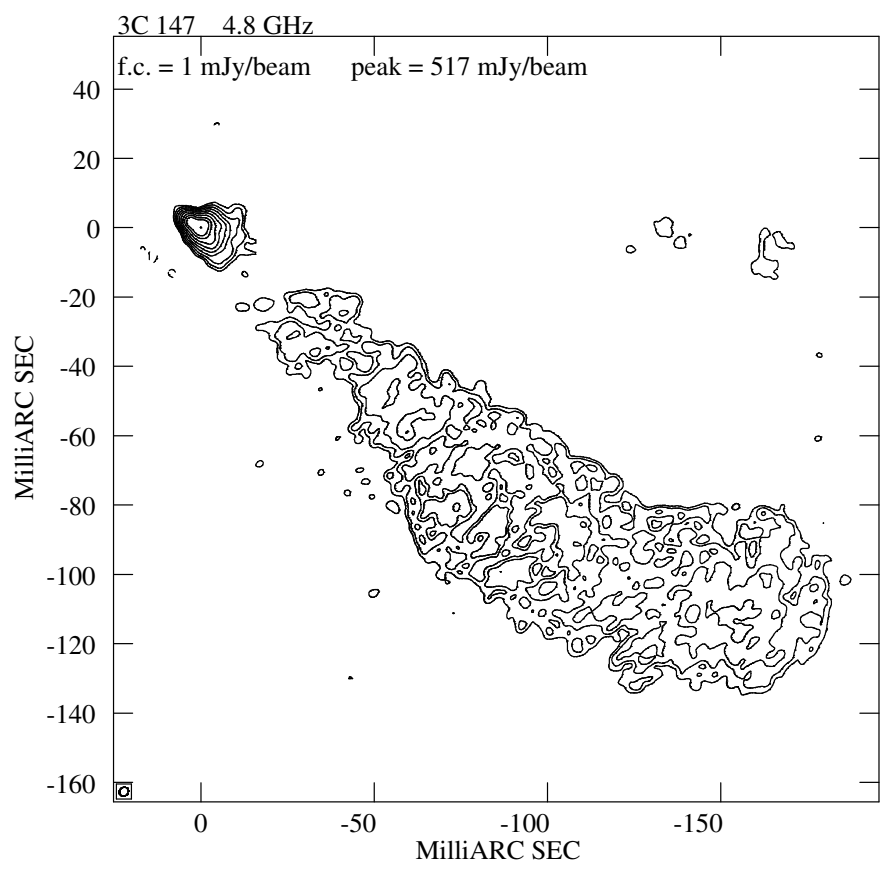

Fig. 1. The total intensity $4.8-\mathrm{GHz}$ image of $3 \mathrm{C} 147$ using all four $C$-band IFs and a restoring beam of $2.8 \times 2.5$ mas at $-40^{\circ}$. Contour levels increase by a factor of 2 from $1 \mathrm{mJy} / \mathrm{beam}$.

contained 4 independent frequencies, namely 4650, 4845, 4853, and $5052 \mathrm{MHz}$, while the $X$-band observations by Zhang et al. (2004) were centred on 7904, 8238, 8562, and $8888 \mathrm{MHz}$. This allows us to determine the rotation measure of the source by using 8 and 5 data points at $C$ - and $X$-band, respectively.

We calibrated the data using the same strategy as described in Sect. 2.1. Images were made with a restoring beam of $2.0 \times$ 1.5 mas at $\mathrm{PA}-6^{\circ}$ and the same $u v$-range sampling as for our data, to perform a pixel-by-pixel comparison. The resultant images look very similar to those obtained from our own data. The total flux densities that we obtained agree (within 3\%) at both $C$ and $X$-bands.

\section{Results}

\subsection{The $C$-band results}

The total-intensity image of $3 \mathrm{C} 147$ at $5.0 \mathrm{GHz}$ obtained using all the four $C$-band frequencies is shown in Fig. 1. This image was tapered to improve the sensitivity to low brightness emission. The off-source rms noise level achieved in this image is about $0.2 \mathrm{mJy} / \mathrm{beam}$, just over a factor of two above the expected thermal noise.

We measured a total flux density of $4.70 \mathrm{Jy}$, which is $57 \%$ of the single-dish flux density (Pauliny-Toth et al. 1978) implying that a large fraction of the flux density was resolved. On the mas scale, 3C 147 shows a one-sided structure with a complex compact region, and a component extending to the southwest, which is visible out to a distance of $\approx 200$ mas from the compact component. This soon broadens without losing its collimation, shows several slight wiggles and, finally, bends to the west close to its end. To compare the 5 and $8.4 \mathrm{GHz}$ results, the images need to be of the same resolution. Ideally, the $u v$-coverages should be identical. However, this is impossible with VLBI arrays. Therefore, images of the central component were made using the $u v$-range between the shortest baseline at $X$-band and the longest baseline at $C$-band and a restoring beam of $2.0 \times 1.5$ mas at PA $-6^{\circ}$. At

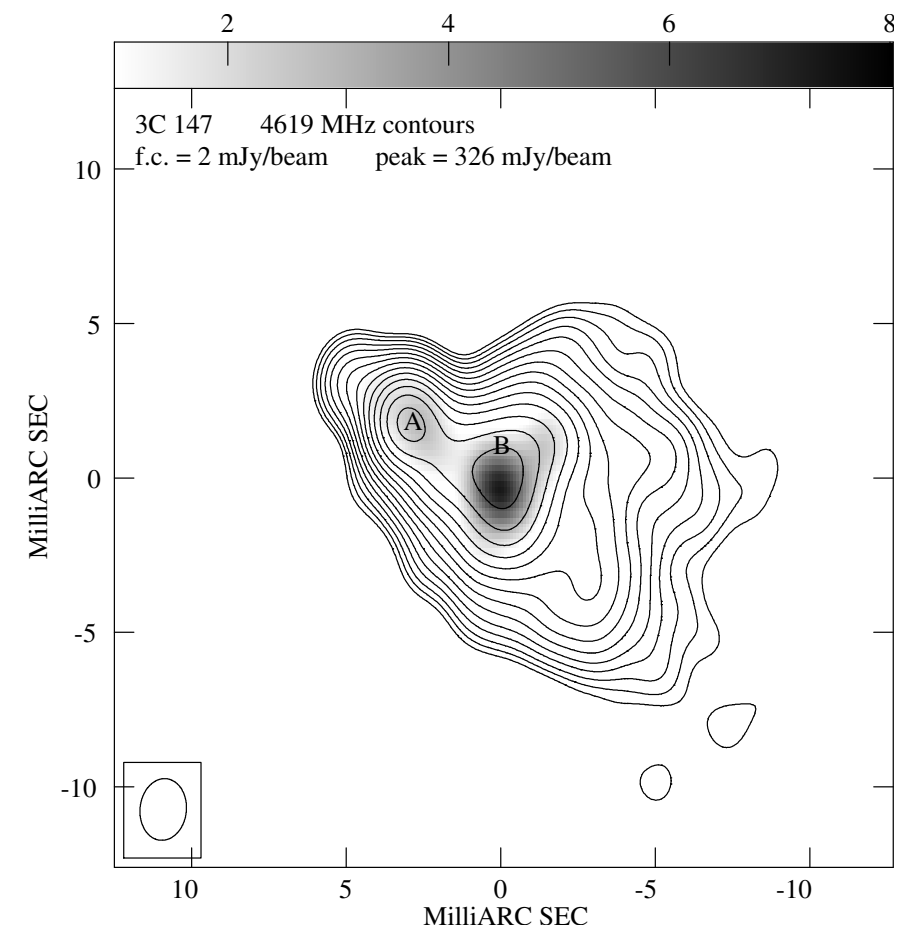

Fig. 2. The grey-scale image of $C$-band polarised intensity with contours of total intensity for IF $1(4619 \mathrm{MHz})$ superimposed. The angular resolution is $2.0 \times 1.5$ mas at $\mathrm{PA}-6^{\circ}$. Contour levels increase by a factor of $\sqrt{2}$ from $2 \mathrm{mJy} /$ beam.

Table 1. Source parameters.

\begin{tabular}{cccccc}
\hline \hline Comp. & $\begin{array}{c}\text { IF } \\
\mathrm{MHz}\end{array}$ & $\begin{array}{c}S \\
\mathrm{mJy}\end{array}$ & $\begin{array}{c}S_{\mathrm{p}} \\
\mathrm{mJy}\end{array}$ & $\begin{array}{c}\chi \\
\circ\end{array}$ & \%pol \\
& 4619 & $335 \pm 10$ & $3.3 \pm 0.3$ & $-7 \pm 5$ & $1.0 \pm 0.1$ \\
$\mathrm{~A}$ & 4657 & $327 \pm 10$ & $3.1 \pm 0.3$ & $-1 \pm 5$ & $0.9 \pm 0.1$ \\
& 4854 & $331 \pm 10$ & $3.0 \pm 0.3$ & $15 \pm 5$ & $0.9 \pm 0.1$ \\
& 5094 & $319 \pm 10$ & $2.9 \pm 0.3$ & $29 \pm 5$ & $0.9 \pm 0.1$ \\
& 8409 & $331 \pm 10$ & $7.1 \pm 0.6$ & $196 \pm 5$ & $2.1 \pm 0.2$ \\
$\mathrm{~B}$ & & & & & \\
& 4619 & $596 \pm 18$ & $8.9 \pm 0.4$ & $-47 \pm 5$ & $1.5 \pm 0.1$ \\
& 4657 & $579 \pm 17$ & $10.5 \pm 0.4$ & $-44 \pm 5$ & $1.8 \pm 0.1$ \\
& 4854 & $587 \pm 18$ & $12.1 \pm 0.4$ & $-14 \pm 5$ & $2.1 \pm 0.1$ \\
& 5094 & $565 \pm 17$ & $10.6 \pm 0.4$ & $+18 \pm 5$ & $1.9 \pm 0.1$ \\
& 8409 & $491 \pm 15$ & $31.7 \pm 1.0$ & $+161 \pm 5$ & $6.5 \pm 0.4$ \\
\hline
\end{tabular}

$C$-band, we made images of the central component for each of the four individual IFs.

Figure 2 shows the structure of the central region for IF 1 (4619 MHz). The image reveals two compact components, A and $\mathrm{B}$, embedded in more diffuse emission. The grey scale represents the distribution of polarised intensity. Polarised emission is detected from both components $\mathrm{A}$ and $\mathrm{B}$, the overall degree of $C$-band polarisation being about $1.0 \%$ and $2.0 \%$ for components A and B, respectively, (see Table 1). Flux densities were determined using the task IMEAN on the same region of the $\mathrm{P}$ and I images. In Table 1, the total flux densities, $S$, the polarised flux densities, $S_{\mathrm{p}}$, the electric vector position angle, $\chi$ and the fractional polarisation, $\%$ pol, are listed for the four $C$-band IFs and for the $X$-band.

\subsection{The $X$-band results}

The full resolution total-intensity image of the central component of 3C 147 obtained using all the contiguous IFs at $8.4 \mathrm{GHz}$ 


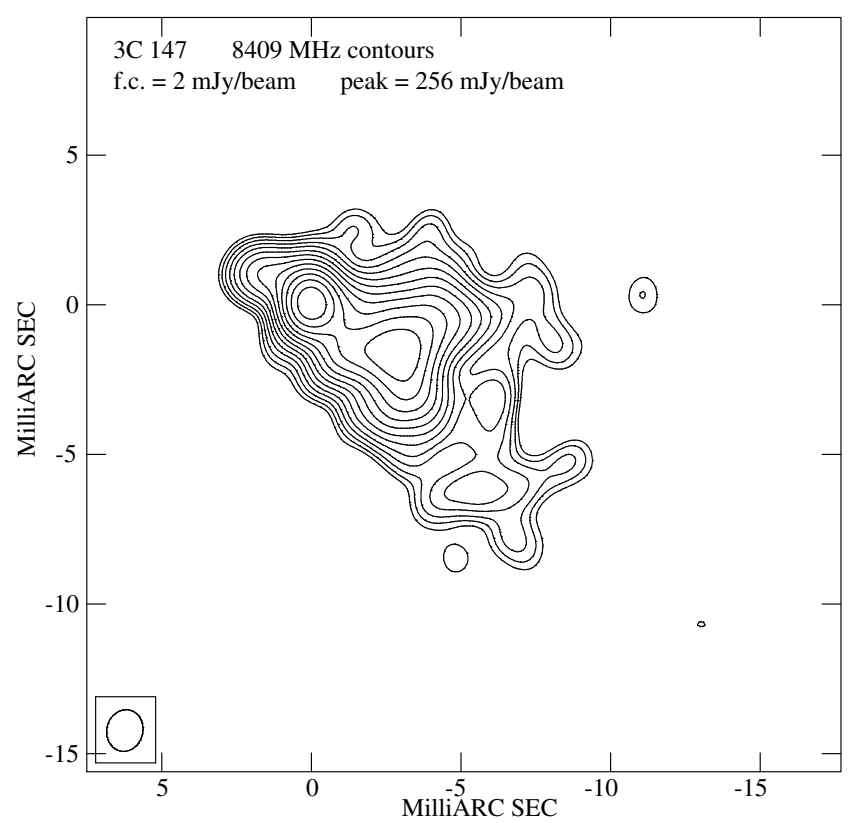

Fig. 3. Total intensity contours of $3 \mathrm{C} 147$ at $8.4 \mathrm{GHz}$ using all four $X$-band IFs and a restoring beam of $1.4 \times 1.2$ mas at PA $-14^{\circ}$. Contour levels increase by a factor of $\sqrt{2}$ from $2 \mathrm{mJy} /$ beam.

is given in Fig. 3. At this resolution the diffuse emission from the outer part of the extended component is completely resolved. The rms noise level in this image is $0.3 \mathrm{mJy} /$ beam and we measured a total flux density of about $1.6 \mathrm{Jy}, \approx 33 \%$ of the single-dish flux density (Rickett et al. 2006).

The structure of the compact component changes little between the $X$ - and $C$-band. In Fig. 3 , the source shows the two compact components $\mathrm{A}$ and $\mathrm{B}$ embedded in a region of diffuse emission as at $C$-band. However, this image has a more complex morphology with two bulges of emission, BN and BS, for component $\mathrm{B}$ and a slightly resolved feature, $\mathrm{A}_{0}$, to the northeast of component A.

The polarised flux density, whose distribution is represented in Fig. 4 in grey scale, reflects the complex structure of the central region. Component $\mathrm{A}$ is polarised at a level of about $2 \%$. The peak of the $X$-band polarised intensity is not coincident with the peak polarised intensity found at $C$-band. This is also seen in other sources that show evidence of $R M$ gradients (e.g., 3C 119; Nan et al. 1999). For component $\mathrm{A}_{0}$, we do not detect polarised emission.

Component B is resolved into two separated polarised regions associated with peaks of emission to the northwest (BN) and to the south (BS). In these two regions, the fractional polarisation reaches values of $6.6 \%$ and $5.6 \%$, respectively.

\subsection{Comparison of $C$ - and $X$-band results}

To compare $C$ - and $X$-band images, they must be aligned with each other. Absolute positional information is lost by the use of self-calibration techniques, and the 5- and 8.4-GHz images were instead aligned relative to each other by forcing the positions of the peak brightness of component $\mathrm{B}$ to be coincident by means of the AIPS task LGEOM.

The spectral index values of the central components were obtained for a 5-frequency data cube using the AIPS task SPIXR.

The spectral indices that we estimated are $\alpha_{5}^{8.4} \approx 0.04$ for component $\mathrm{A}$ and $\alpha_{5}^{8.4} \approx 0.31$ for component $\mathrm{B}$. The

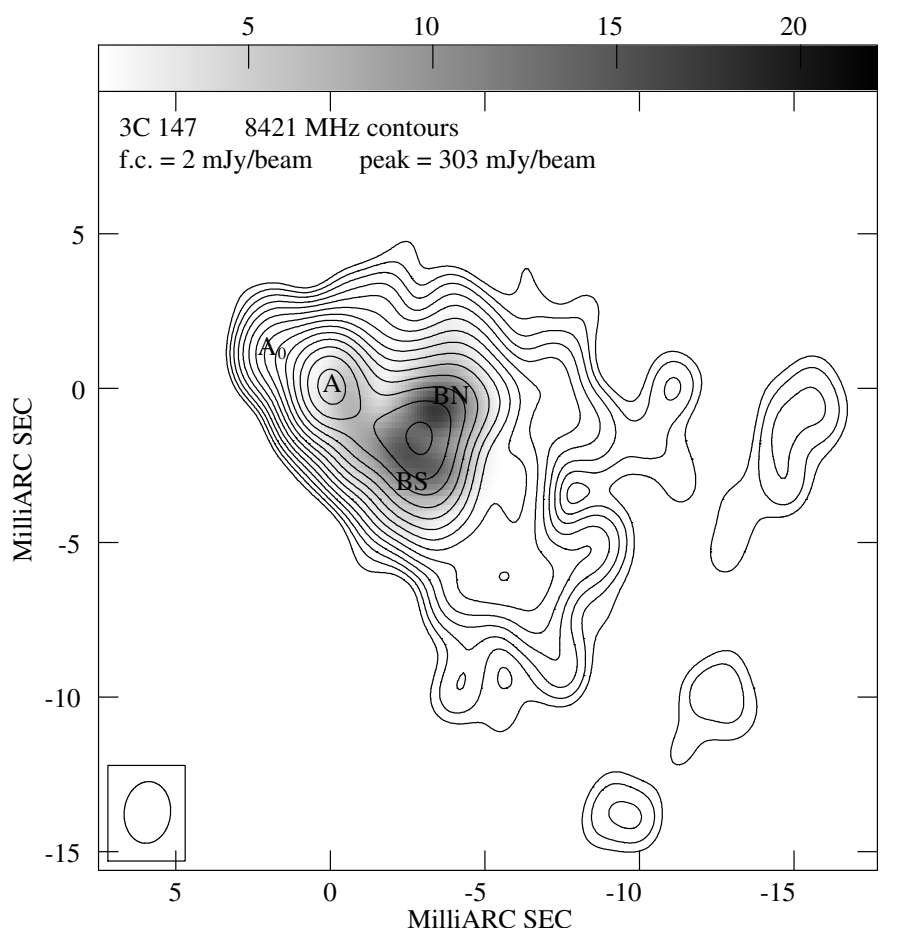

Fig. 4. Total intensity contours at $8421 \mathrm{MHz}$ with a grey-scale image of polarised intensity superimposed. The angular resolution for both total and polarised intensities is as for Fig. 2. Contour levels increase by a factor of $\sqrt{2}$ from $2 \mathrm{mJy} / \mathrm{beam}$.

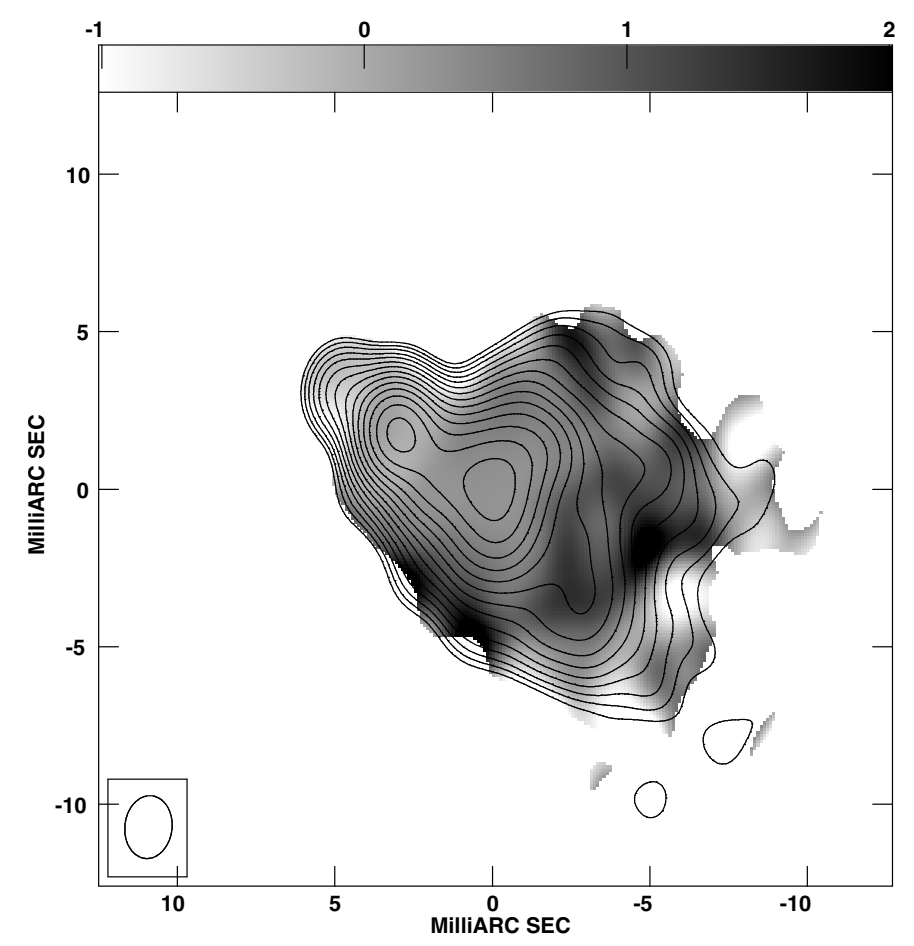

Fig. 5. A grey-scale image of the spectral-index distribution with the total-intensity contours for $C$-band IF $1(4619 \mathrm{MHz})$ superimposed. Contour levels increase by a factor of $\sqrt{2}$ from $2 \mathrm{mJy} / \mathrm{beam}$.

northeastern, slightly resolved component, $\mathrm{A}_{0}$, has an inverted spectral index $\alpha_{5}^{8.4} \approx-0.4$. The overall spectral-index distribution is displayed in Fig. 5 in grey-scale. 


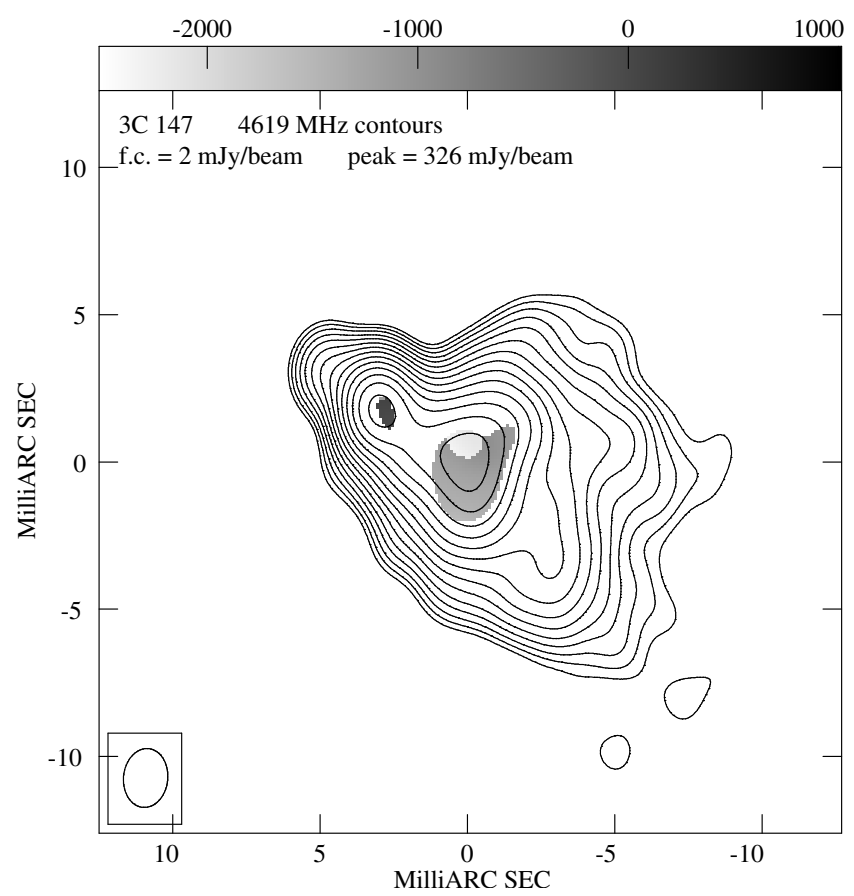

Fig. 6. Total-intensity $C$-band contours for IF 1 (4619 MHz) with the $R M$ distribution superimposed in grey scale.

\subsection{Rotation measure structure and intrinsic magnetic field}

Figure 6 shows in grey-scale the $R M$ distribution for $3 \mathrm{C} 147$ across four frequencies overlaid on the total-intensity contour image. No redshift corrections were applied to the wavelengths, so the $R M$ in the rest frame of the source will be higher by a factor of $(1+z)^{2} \approx 2.4$. The $R M$ distribution is not uniform but exhibits structure. The highest $R M \mathrm{~s}$ are associated with the northern polarised region of component $\mathrm{B}$. The distribution of intrinsic magnetic-field orientation is shown in Fig. 7. The magnetic-field structure is complex and in the central component is perpendicular to the major axis of the source.

As mentioned in Sect. 1, the behaviour of the electric vector polarisation angle, $\chi$, as a function of $\lambda^{2}$ allows us to obtain information about the density distribution of the ionised ISM around the radio source. For a homogeneous (both in density and magnetic field) medium or a medium with inhomogeneities resolved by the observing beam, the polarisation angle is strictly proportional to $\lambda^{2}$ at all wavelengths. For an unresolved or partially resolved medium, the $R M$ changes from point to point across the source, different contributions of polarised radiation are rotated differently, and the polarisation angle can deviate from the $\lambda^{2}$-linear law.

Figure 8 presents the polarisation angle of components $\mathrm{A}$ (open circles) and B (filled circles) as a function of $\lambda^{2}$. Among the four $C$-band IFs, we found a good linear fit with $R M_{\mathrm{A}}=$ $-811 \pm 62 \mathrm{rad} \mathrm{m}^{-2}$ and $R M_{\mathrm{B}}=-1544 \pm 73 \mathrm{rad} \mathrm{m}^{-2}$. However, the $8.4-\mathrm{GHz}$ data points are offset by about $+64^{\circ}$ and $-45^{\circ}$, respectively.

To investigate the $R M$ structure of 3C 147 and the deviation of the electric vector polarisation angle from the $\lambda^{2}$-law further, we searched the VLBA data archive for available data to enable a direct comparison with our observations.

\subsection{Comparison of observations and archival data results}

The direct comparison between our images and those made from archival data allows us to derive some interesting results.

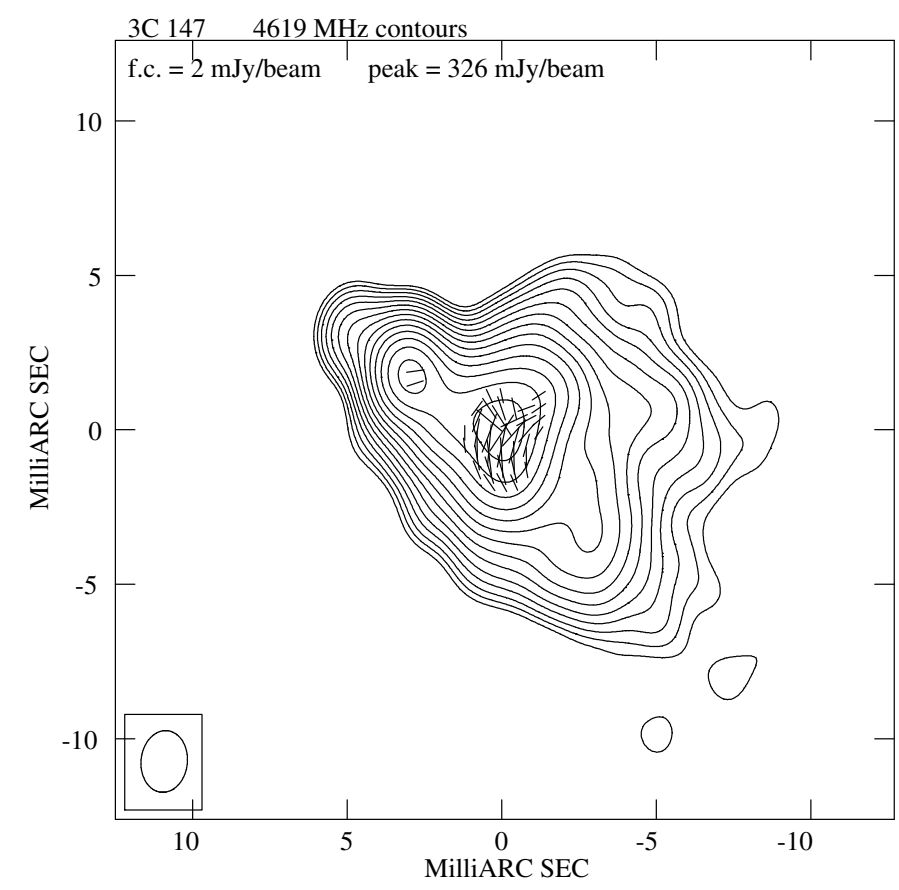

Fig. 7. Total-intensity $C$-band contours for IF 1 (4619 MHz) with the intrinsic orientation of the projected $\boldsymbol{B}$-field superimposed.

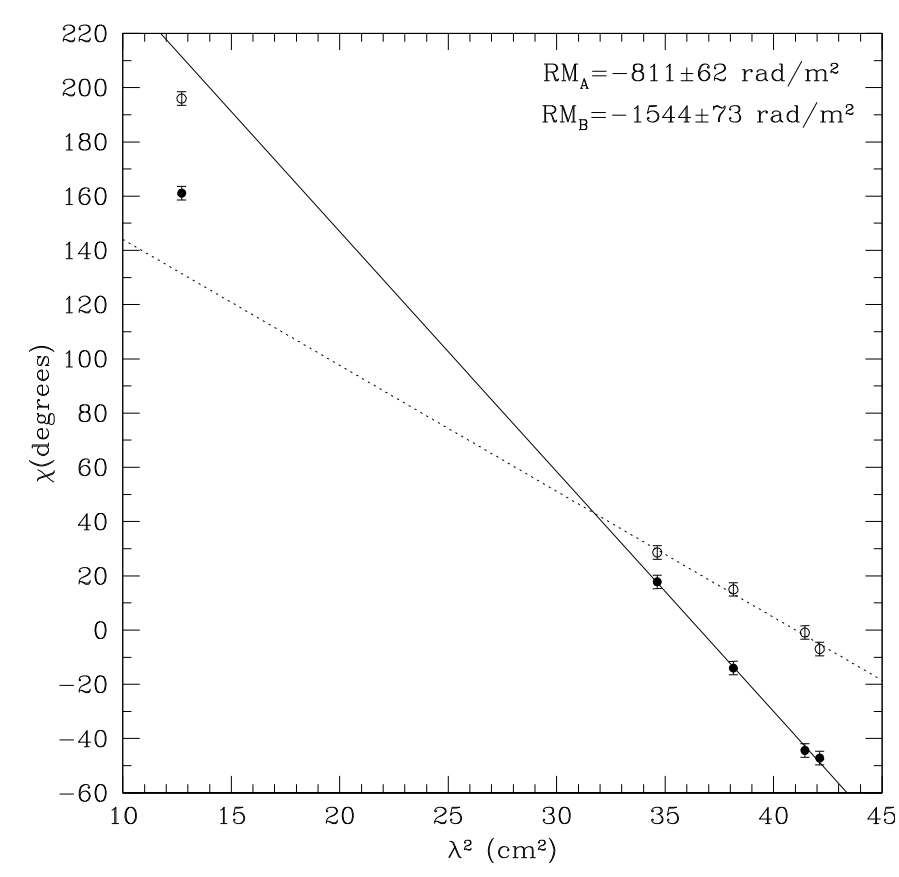

Fig. 8. A plot of the observed $\chi$ values for components A (open circles) and $\mathrm{B}$ (filled circles) as a function of $\lambda^{2}$ for the five available wavelengths.

Aller et al. (2003) reported small systematic changes in flux density on a timescale of several years. They suggested that the central region is responsible for this intensity variability and also found variability in the integrated polarised flux density.

At $C$-band, we found that the total flux density we measured in our images is consistent with the flux density measured in images from project $\mathrm{BC} 033$, within the calibration uncertainties. However, between the two observing epochs there are significant 


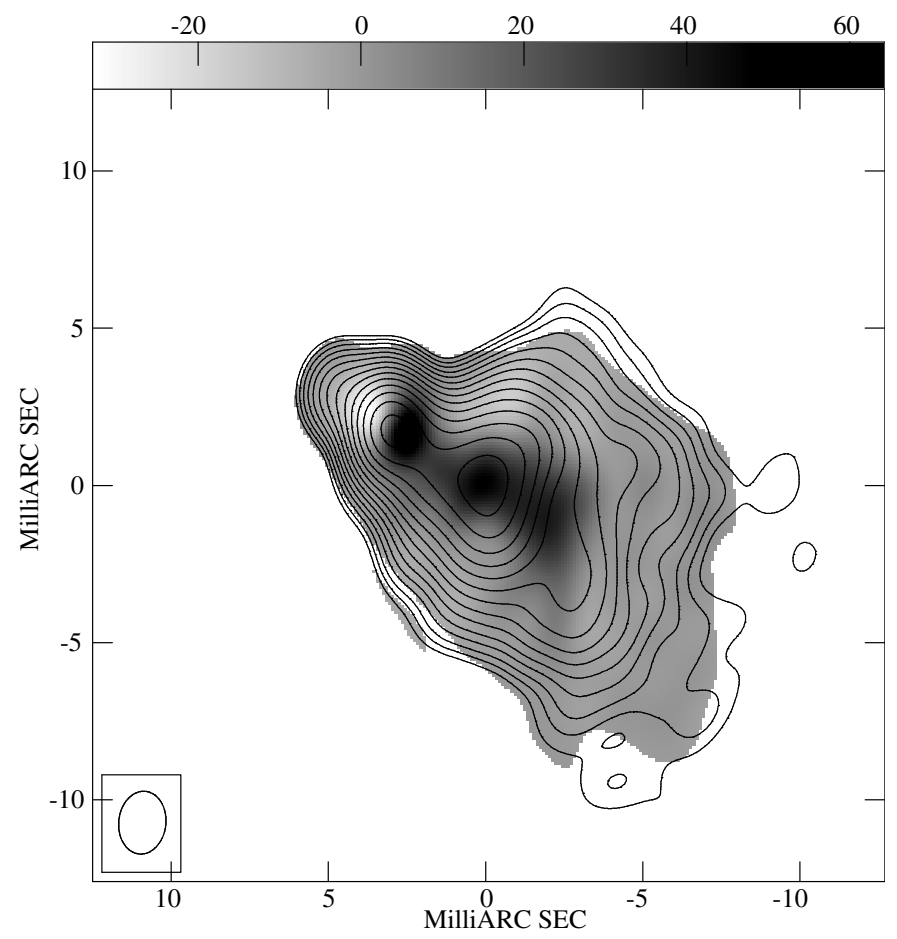

Fig. 9. A grey-scale image of the distribution of the $C$-band, total-intensity differences between 1995 and 2001, with contours of total intensity for IF 3 (4854 MHz) superimposed. Contour levels increase by a factor of $\sqrt{2}$.

differences in the physical properties of the resolved components that we summarise as follows:

- we imaged the difference in total-intensity between the 1995 data from project BC033 (Nan et al. 2000), and our data at the same frequency, i.e., for IF 3 of each data set (Fig. 9). The peak brightnesses of component $\mathrm{A}$ at the two epochs are not coincident. Since the images were aligned by the position of component $\mathrm{B}$, this indicates that the separation between components $\mathrm{A}$ and $\mathrm{B}$ has increased;

- in Nan et al. (2000), there were indications that component $\mathrm{A}$ is not polarised (with an upper limit to the fractional polarisation of about $0.5 \%$ ), and we confirm this result for images that we made from their data. Based on the lack of polarised emission, they identified component A with the core. However, from our images of the epoch 2001 data, we measured a polarised flux density associated with component A of about $3 \mathrm{mJy}$ (see Table 1), with a noise level of $0.4 \mathrm{mJy} /$ beam, i.e., a factor of eight above the rms, implying the presence of polarised emission at the $1 \%$ level. Therefore, we inferred a significant increase in the $C$-band fractional polarisation associated with component A between 1995 and our observing epoch of 2001.

Component B does not show any changes in its polarisation properties between 1995 and 2001, and the electric vector angles derived for the two data sets at $C$-band are in good agreement, giving a local $R M$ of $-1504 \pm 58 \mathrm{rad} \mathrm{m}^{-2}$. Figure 10 shows the electric vector polarisation angle as a function of $\lambda^{2}$ at $C$-band for component $\mathrm{B}$ as a whole by using the four IFs from project $\mathrm{BC} 033$ (open circles) and the four IFs from our data (filled circles).

At $X$-band, we found no substantial differences between our data and data from project BZ0023 (Zhang et al. 2004) for both components. Since these observations were made at similar

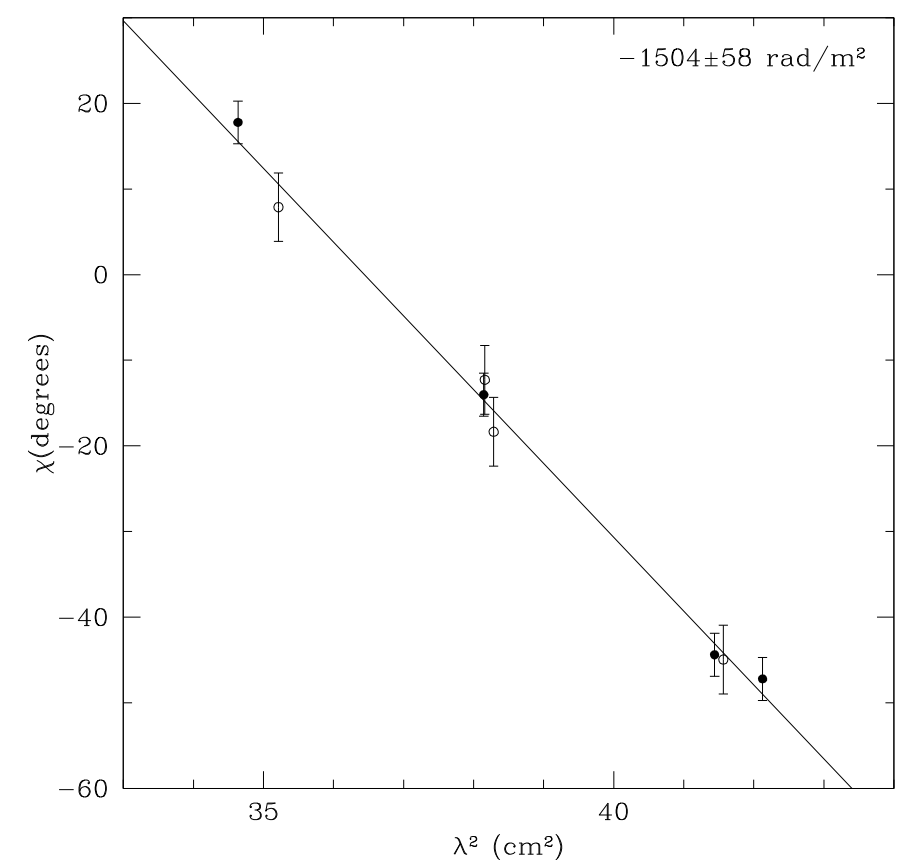

Fig. 10. A plot of the observed $\chi$ values for component $B$ as a function of $\lambda^{2}$. Filled circles denote the four $C$-band IFs of our observations, while the open circles are the four $C$-band IFs from Nan et al. (2000).

epochs (May 2001 and Aug. 2000), this gives us confidence in the reality of the $C$-band changes detailed above.

In Fig. 11, we show the position angle of the electric vectors as a function of $\lambda^{2}$ for the two polarised regions in component $\mathrm{B}$. The plots were obtained by using data from project BZ0023 (open circles) and our single data point (filled circle). These are fitted rather well with $R M$ s of $-1457 \pm 195 \mathrm{rad} \mathrm{m}^{-2}$ and $-2041 \pm 195 \mathrm{rad} \mathrm{m}^{-2}$ for the features to the south and north respectively. Within the errors produced by noise and fitting to the polarisation position angles, these $R M$ values agree with the single-dish measurements by Inoue et al. (1995). Therefore, the compact region can be considered to be the major component responsible for the high integrated $R M$ of this radio source.

\subsection{Relativistic motion}

Nan et al. (2000) found indications of an increase in the separation between components A and B. By comparing their data with the results of Alef et al. (1990), they found an apparent speed of $\approx 1.5 c$. To investigate this separation further, we analysed the position of the components by fitting a Gaussian to each component (in images obtained by using a selected range of visibilities to sample the compact components only and a restoring beam of $1 \times 1$ mas) and determining the distance between the peaks of $\mathrm{A}$ and $\mathrm{B}$. We found that the separation between the two components increased by $0.6 \pm 0.2$ mas between May 1995 and May 2001, as measured from the 5-GHz images for which the separation of epochs was the largest. This corresponds to a relative motion between components A and B with an apparent speed of expansion of $1.2 \pm 0.4 c$.

\section{Discussion}

\subsection{Interpretation of the $\lambda^{2}$ linear law deviation}

The deviation from a $\lambda^{2}$ law observed in component $\mathrm{B}$ between $C$ - and $X$-band (Fig. 10) can be explained in terms of the 

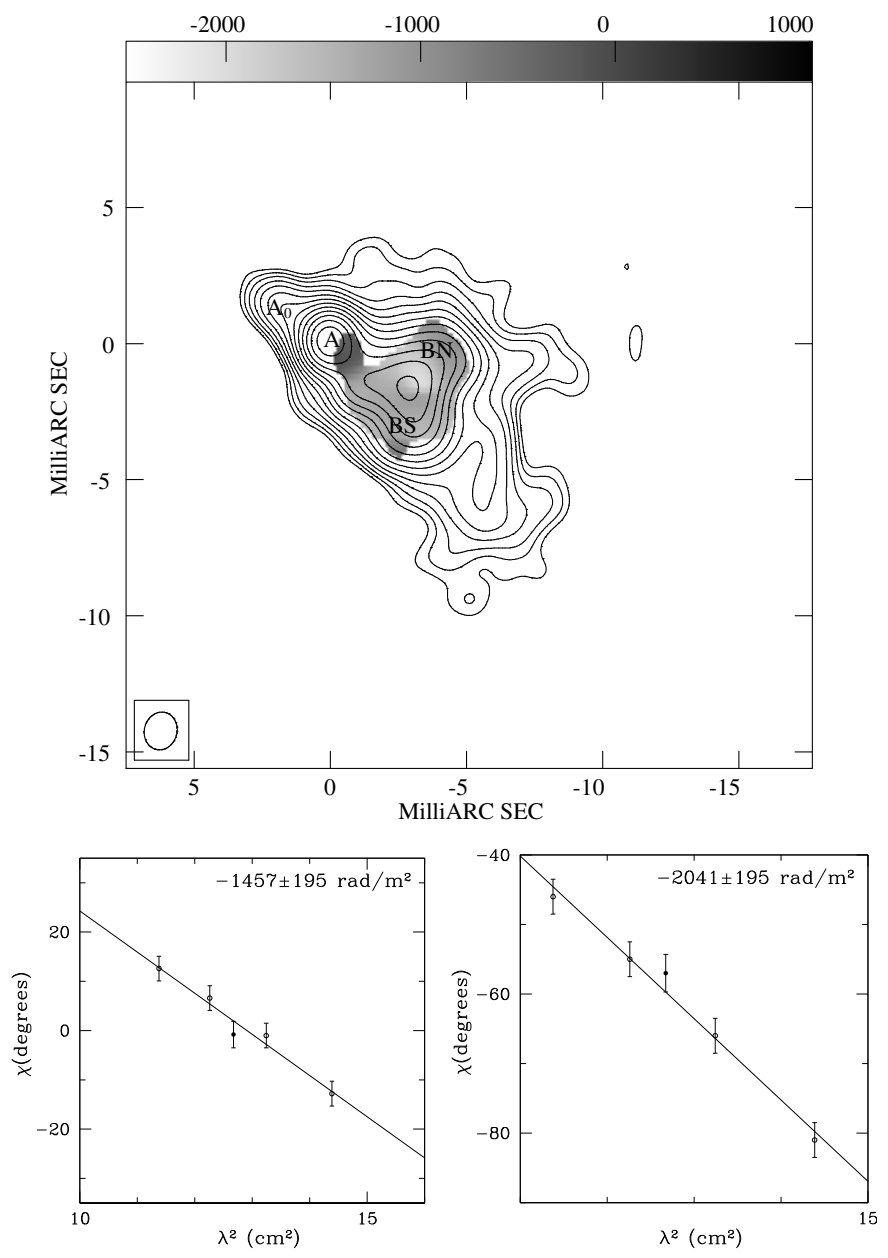

Fig. 11. Contours of $X$-band total intensity, with the $R M$ distribution from project BZ0023 (Zhang et al. 2004) superimposed in grey scale. The two inserts show plots of $\chi$ versus $\lambda^{2}$ for the four IFs of the Zhang observation and our single $X$-band data point for the two polarised regions in component $\mathrm{B}$, (the southern component to the left, and the northern component to the right).

contributions of two or more polarised components with different $R M$ s. Component B clearly shows evidence of two compact polarised regions with different $R M$ s (see Figs. 4 and 11). Therefore, at $8.4 \mathrm{GHz}$, the polarisation angle that we are accounting for is an "average value". Neglecting other effects, it equals the angle of the vector $\boldsymbol{P}$ obtained by the vectorial sum of the two contributions. In such a situation, the polarisation angle is not expected to follow a $\lambda^{2}$ law (Rossetti et al. 2008). An analogous interpretation could also explain the deviation from the $\lambda^{2}$ law observed in component $\mathrm{A}$, although this component was not resolved into sub-components by the present observations.

\subsection{Possible explanations of the source structure}

\subsubsection{Scenario 1: the central region of $3 C 147$ harbours the core and jet}

The standard interpretation of the radio structure of $3 \mathrm{C} 147$ is that the central bright radio-emitting region contains the source nucleus. This scenario is supported by the following evidence:

- The presence of two bright compact components, one with a flat spectrum (A) between 5 and $8 \mathrm{GHz}$, can be interpreted as a core plus a bright knot in the jet (Nan et al. 2000).

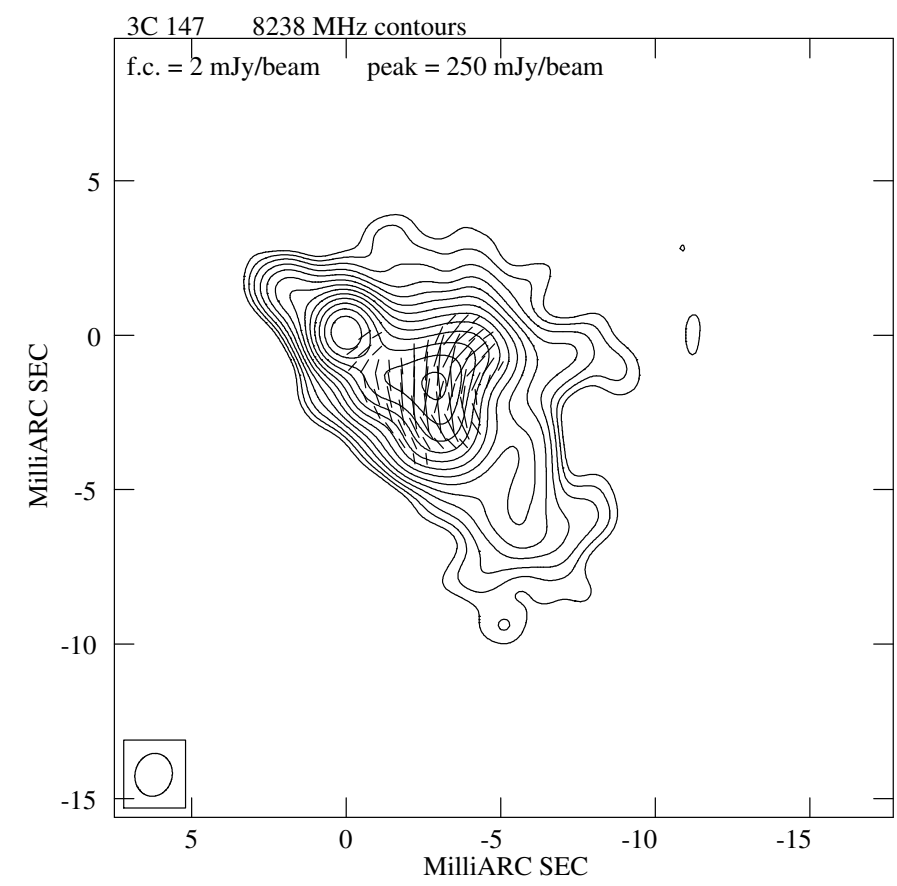

Fig. 12. Total intensity contours at $8238 \mathrm{MHz}$ with the intrinsic orientation of the projected $\boldsymbol{B}$-field overlaid.

Lüdke et al. (1999) and Zhang et al. (2004) detected an additional slightly resolved component to the northeast of component $\mathrm{A}$, and, based on its weak polarisation and its position at one end of the source structure, they identified it to be the true core. If the core has lower polarisation than the knots, as found in core-dominated quasars (Cawthorne et al. 1993), milliarcsecond-resolution polarisation images should be very useful in identifying the core in $3 \mathrm{C} 147$. However, although the northeast component $\mathrm{A}_{0}$ is not completely resolved by the available observations, Zhang et al. (2004) estimated its flux density to range from 32 to $26 \mathrm{mJy}$ at 7904 and $8888 \mathrm{MHz}$ respectively. The peak polarised signal is less than $1 \mathrm{mJy} /$ beam, suggesting that the degree of polarisation is less than $\approx 3 \%$.

- The triple structure found at subarcsecond resolution, which suggests the existence of a compact central core in-between two extended, asymmetric lobes, which are probably expanding within different environments (Junor et al. 1999a).

It is important to assess whether this scenario is consistent with the magnetic field orientation along the axis of the jet (Fig. 12).

Although early work suggested that in quasars, the magnetic field is often parallel to the jet axis (Cawthorne et al. 1993; Dallacasa et al. 1995), more recent surveys identified a more complex situation. For example, from polarimetric observations of 177 sources from the Caltech-Jodrell Bank Flat-Spectrum Survey, Pollack et al. (2003) found no evidence of field direction alignment with the jet axis. Similar conclusions were reached by Lister \& Homan (2005) based on milliarcsecond-scale linear polarisation observations at $15 \mathrm{GHz}$ of 133 jets associated with active galactic nuclei. Field lines on parsec scales can be complex in radio jet-ambient medium interactions, as seen, for example, in the quasar B1055 + 018 (Attridge et al. 1999). Therefore, the magnetic field orientation in the compact region of $3 \mathrm{C} 147$, where there is possibly strong jet-medium interactions, would not be unusual.

However, the identification of the source core still remains questionable. Observation of far higher resolution were carried 
out by Lister (2001) at $43 \mathrm{GHz}$ for the central region of 3C 147. A bright, compact source of size about 0.25 mas with a flux density of $177 \mathrm{mJy}$ and an upper limit to the degree of polarisation of $3.2 \%$, was detected by these observations. However, this component cannot be clearly identified with any of the features found at lower frequencies within the source. It may correspond to either of our components $\mathrm{A}$ or $\mathrm{A}_{0}$; its measured flux density is compatible with the flat spectrum found for component $\mathrm{A}$ between 5.0 and $8.4 \mathrm{GHz}$. Although component A shows polarised emission, its value is consistent with the measurements of quasar core polarisation, for which values can be as high as $10 \%$, but the typical value is approximately a few per cent (Pollack et al. 2003; Lister \& Homan 2005).

On the other hand, if $\mathrm{A}_{0}$ is identified with the component seen at $43 \mathrm{GHz}$ by Lister (2001), it would have an inverted spectrum with a spectral index of approximately -1 . This would be consistent with the most inverted spectrum component, located at one end of the jet, being the core, as also seen in $3 \mathrm{C} 48$ (Wilkinson et al. 1991). However, the spectrum should turn over so that the extrapolated flux density at $230 \mathrm{GHz}$ does not exceed the total flux density value of $280 \pm 28 \mathrm{mJy}$ determined by Steppe et al. (1995).

\subsubsection{Scenario 2: the compact region of $3 \mathrm{C} 147$ is a hot spot complex}

A second interpretation that we discuss for the radio structure of $3 \mathrm{C} 147$ is that the brightest region imaged in Fig. 2 is the approaching hot spot of a powerful radio source, whose main axis is quite close to the line of sight. The hot spot is marginally Doppler boosted, and its structure, with multiple, individual knots, is similar to the multiple hot spots found in several powerful double radio galaxies when studied with appropriate resolution. What was previously defined as a "jet" is now interpreted as the backflow tail of the approaching lobe. The other lobe lies about 1'.2 to the north (Junor et al. 1999a) and its hot spot is Doppler dimmed, since particle acceleration takes place on its receeding side. The strong asymmetry between the two lobes in both size and flux density may arise from differing environments, as suggested by Junor et al. (1999a). In that case, the main lobe appears brighter and smaller because of stronger confinement of the radio-emitting plasma. If the brightest lobe is confined by a far denser medium, we would expect ionization of the ambient medium in the region where interaction with the radio jet takes place. This would explain the high Faraday rotation that we observe.

The overall properties of $3 \mathrm{C} 147$ are similar to those found in 3C 286 (Jiang et al. 1996; Cotton et al. 1997), and possibily 3C 138 (Cotton et al. 2003b) (although both of these sources are highly polarised with low/absent $R M$ ) whose interpretation presents similar challenges.

Evidences that are consistent with this lobe-hot spot scenario can be summarised as follows:

- The polarisation properties (magnetic field topology and fractional polarisation) in the brightest region of the source are consistent with a hot spot of multiple structures.

- The extended structure to the southwest of the brightest region, which we propose to be a backflow tail, has a smooth brightness distribution; the structure is rather slim and is similar to the lobes in thin FR II sources, such as 3C 284 and 3C 341. On the small scale, it resembles the main "jet" of 3C 138 (e.g., Cotton et al. 2003b), which is not a jet, since the current interpretation of the source postulates that the easternmost and brightest structure is indeed a hot spot.

- The size of the various components and the width-to-length ratio of the brightest region can be more easily reconciled with a hot spot rather than the initial part of a jet (i.e., the core), since this would require substantial recollimation to maintain a small opening angle along the entire structure to the southeast. We note that there are some examples of jets exhibiting large opening angles and later recollimating, as seen in M 87 (Junor et al. 1999b) and, to some extent, in B1055 + 018 (Attridge et al. 1999), even if this was found to occur on far smaller scales.

However, a few aspects still require explanation because they do not fully agree with this "hot spot" interpretation.

\section{- The core has yet to be found.}

Among the various possibilities, the small-scale jet axis may be misaligned with the large-scale jet and, in particular, be so close to the plane of the sky that the highly relativistic jets are Doppler dimmed, or the radio activity may have recently been switched off, as proposed by KunertBajraszewska et al. (2005) for the "fader" radio sources. The hot spots remain active, while the plenishment of freshly created particles has ceased.

Alternatively, a "normal" weak (steep-spectrum?) core appears to be embedded within the diffuse emission of the lobe+hot spot complex, and cannot be clearly identified in present observations. Its contribution to the total flux density of the radio source at $\mathrm{cm}$ wavelengths would be marginal, given that the source is known to be quite stable in flux density.

- The northern lobe is difficult to explain if we consider a model of a small-sized classical double structure, such as Cygnus A, seen in projection. However, there are powerful sources in which radio plumes emerge from the classical double structure, and 3C 147 may resemble either 3C 249.1 or 3C 351 seen along the source major axis.

- Superluminal motion has been observed between the two compact components A and B. The apparent separation speed of the knots in the compact component of 3C 147 (see Sect. 3.6) is consistent with the general model of AGN radio sources, in which the jet is assumed to be initially highly relativistic. In case of a jet termination along the line of sight, it might be possible to observe relative separation at the apparent speed of light.

\subsection{Comparison with other polarised CSS quasars}

Only a minority of CSS quasars has been imaged so far with polarimetric VLBI observations. As an example, data have been published for only 7 of the 24 objects in the list of CSSs from the 3C and PW catalogues (Fanti et al. 1990). All of these show a core-jet structure. Polarised emission is detected along the jet, showing fractional polarisations of between $2 \%$ and $20 \%$. In contrast to flat spectrum quasars the cores, when detected, are not polarised. CSSs for which mas-scale $R M$ distributions have been derived are even rarer. We note here the cases of OQ 172 (Udomprasert et al. 1997), 3C 216 (Venturi \& Taylor 1999), $0548+165$ and $1524-136$ (Mantovani et al. 2002), 3C 43 and 3C 454 (Cotton et al. 2003c), and 3C 138 (Cotton et al. 2003b). In those cases, rest-frame $R M>10^{3} \mathrm{rad} \mathrm{m}^{-2}$, and even as high as $2 \times 10^{4}$ are found. The fractional polarisation decreases moving from higher to lower frequencies. Generally speaking, the $R M$ is 
explained in terms of a foreground Faraday screen, where a NLR sometimes contributes to the $R M$. The intensity of the magnetic field is estimated to be a few tenths of a $\mu$ Gauss. Jets are often distorted and this can also be interpreted in terms of jet-cloud interactions or projection effects.

The images of 3C 147 presented here show a relatively undisturbed "jet", which has only a few slight wiggles and no sharp turns and distortions seen in many other CSS sources. The only bend visible in our images is at the end of the component to southwest. The observed high $R M$ values are associated with the compact region, while in many core-jet CSS quasars high integrated Faraday rotation takes place in the bright region where the bend occurs.

\section{Summary and conclusions}

By analysing new polarimetric observations at $C$ - and $X$-band, and re-analysing previous VLBA observations, we have been able to image the milliarcsecond structure of the quasar 3C 147. We have identified a complex central region that is dominated by two bright components, $\mathrm{A}$ and $\mathrm{B}$, with a weak, compact component to the northeast, $\mathrm{A}_{0}$, and a further extended component to the southwest, which is imaged out to a distance of 200 mas.

Polarised emission has been detected for both of the compact components, $\mathrm{A}$ and $\mathrm{B}$, at $C$ - and $X$-bands. There are indications of polarised flux-density variability in component $\mathrm{A}$ on a timescale of several years. The polarised emission from component B divides into two independent regions in our observations. The two compact components also show very high values of $R M$ in the rest frame of the source, these being consistent with the rest-frame value reported for the entire main component by Junor et al. (1999a) of $R M=-3140 \pm 630 \mathrm{rad} \mathrm{m}^{-2}$.

The intrinsic orientation of the magnetic field in the central region of $3 \mathrm{C} 147$ follows an arc-shape structure at the southern end of the polarised region. The magnetic-field orientation is almost perpendicular to the source major axis. Similar results were obtained by Junor et al. (1999a) at sub-arcsecond resolution. The separation between the two central components of the source seems to be increasing with an apparent velocity of $1.2 \pm 0.4 c$.

A straightforward interpretation of all the observational data for this radio source is not yet possible. We have discussed the possible location of the core and explored two possible scenarios: a core-jet source, and a lobe-hot spot structure. Further polarisation images, and a more thorough search for the source core, should help us to discriminate between these two scenarios.

Acknowledgements. The VLBA is operated by the US National Radio Astronomy Observatory which is a facility of the National Science Foundation operated under a cooperative agreement by Associated Universities, Inc. We are very grateful to the referee for very helpful comments and suggestions and for a careful reading of the manuscript of this paper.

\section{References}

Akujor, C. E., \& Garrington, S. T. 1995, A\&AS, 112, 235

Alef, W., Preuss, E., \& Kellermann, K. I. 1990, in Proc. Dwingeloo Workshop on Compact Steep Spectrum and GHz Peaked Spectrum Radio Sources, ed. C., Fanti, R., Fanti, \& R. T., Schilizzi, Istituto di Radioastronomia, Bologna, 149

Aller, M. F., Aller, H. D., \& Hughes, P. A. 2003, ApJ, 586, 33

Attridge, J. M., Roberts, D. H., \& Wardle, J. F. C. 1999, ApJ, 518, L87

Axon, D. J., Capetti, A., Fanti, R., et al. 2000, AJ, 120, 2284

Cawthorne, T. V., Wardle, J. F. C., Roberts, D. H., \& Gabuzda, D.C. 1993, ApJ, 416, 519

Cotton, W. D., Fanti, C., Fanti, R., et al. 1997, A\&A, 325, 479

Cotton, W. D., Dallacasa, D., Fanti, C., et al. 2003a, PASA, 20, 12

Cotton, W. D., Dallacasa, D., Fanti, C., et al. 2003b, A\&A, 406, 43

Cotton, W. D., Spencer, R. E., Saikia, D. J., \& Garrington, S. 2003c, A\&A, 403, 537

Dallacasa, D., Cotton, W. D., Fanti, C., et al. 1995, A\&A, 143, 329

Fanti, R., Fanti, C., Schilizzi, R. T., et al. 1990, A\&A, 231, 333

Fanti, C., Fanti, R., Dallacasa, D., et al. 1995, A\&A, 302, 317

Fanti, C., Branchesi, M., Cotton, W. D., et al. 2004, A\&A, 427, 465

Gelderman, R., \& Whittle, M. 1994, ApJS, 91, 491

Inoue, M., Tabara, H., Kato, T., \& Aizu, K. 1995, PASJ, 47, 725

Jiang, D. R., Dallacasa, D., Schilizzi, R. T., et al. 1996, A\&A, 312, 380

Junor, W., Salter, C. J., Saikia, D. J., Mantovani, F., \& Peck, A.B. 1999a, MNRAS, 308, 955

Junor, W., Biretta, J. A., \& Livio, M. 1999b, Nature, 401, 891

Kunert-Bajraszewska, M., Marecki, A., Thomasson, P., \& Spencer, R. E. 2005, A\&A, 440, 93

Lister, M. L. 2001, ApJ, 562, 208

Lister, M. L., \& Homan, D. C. 2005, AJ, 130, 1389

Lüdke, E., Garrington, S. T., Spencer, R. E., et al. 1998, MNRAS, 299, 467

Lüdke, E., Cotton, W. D., Dallacasa, D., \& Sanghera, H. S. 1999, ASPC, 175, 335

Mantovani, F., Junor, W., Fanti, R., Padrielli, L., \& Saikia, D. J. 1994, A\&A, 292, 59

Mantovani, F., Junor, W., Ricci, R., et al. 2002, A\&A, 389, 58

Nan, R. D., Gabuzda, D. C., Kameno, S., et al. 1999, A\&A, 344, 402

Nan, R. D., Zhang, H. Y., Gabuzda, D. C., et al. 2000, A\&A, 357, 891

Pauliny-Toth, I. I. K., Witzel, A., Preuss, E., et al. 1978, AJ, 83, 451

Pollack, L. K., Taylor, G. B., \& Zavala, R. T. 2003, ApJ, 589, 733

Rickett, B. J., Lazio, T. J. W., \& Ghigo, F. D. 2006, ApJS, 165, 439

Rossetti, A., Fanti, C., Fanti, R., Dallacasa, D., \& Stanghellini, C. 2006, A\&A, 449, 49

Rossetti, A., Dallacasa, D., Fanti, C., Fanti, R., \& Mack, K.-H. 2008, A\&A, 487, 865

Saikia, D. J., \& Gupta, N. 2003, A\&A, 405, 499

Saikia, D. J., Swarup, G., \& Kodali, P. D. 1985, MNRAS, 216, 385

Saikia, D. J., Singal, A. K., \& Cornwell, T. J. 1987, MNRAS, 224, 379

Saikia, D. J., Jeyakumar, S., Salter, C. J., et al. 2001, MNRAS, 321, 37S

Sanghera, H. S., Saikia, D. J., Lüdke, E., et al. 1995, A\&A, 295, 629

Spinrad, H., Marr, J., Aguilar, L., \& Djorgovski, S. 1985, PASP, 97, 932

Steppe, H., Jeyakumar, S., Saikia, D. J., \& Salter, C. J. 1995, A\&AS, 113, 409

Udomprasert, P. S., Taylor, G. B., Pearson, T. J., \& Roberts, D. H. 1997, ApJ, 483L, 9

Venturi, T., \& Taylor, G. B. 1999, AJ, 118, 1931

Wilkinson, P. N., Tzioumis, A. K., Benson, J. M., et al. 1991, Nature, 352, 313

Zhang, H. Y., Gabuzda, D. C., Nan, R. D., \& Jin, C. J. 2004, A\&A, 415, 477 